\title{
0 aluno com deficiência em uma perspectiva multidimensional
}

\section{The desability student in a multidimensional perspective}

\begin{abstract}
Marília Costa Dias é pedagoga pela Universidade Federal do Rio Grande do Sul (UFRGS) e mestre em Educação pela Faculdade de Educação da Universidade de São Paulo (FEUSP). Atualmente é professora no curso de Pedagogia do Instituto Superior de Educação Vera Cruz e doutoranda no Programa de Pós-Graduação em Educação da FEUSP.
\end{abstract}

Contato: costadias.marilia@gmail.com

\section{Resumo}

0 artigo discute as concepções de deficiência expressas em documentos de referência da atualidade e suas implicações para a escola a partir de uma reflexão sobre o processo de afirmação dos direitos das pessoas com deficiência. A perspectiva multidimensional coloca o foco nas relações entre os aspectos individuais e o ambiente no qual a pessoa com deficiência está inserida, resultando numa abordagem que dá ênfase às dificuldades funcionais, de modo a favorecer a oferta de serviços e apoios para atender às demandas específicas de cada pessoa. Essa perspectiva tem implicações na elaboração e na implementação de políticas públicas em diversas áreas, e também na educação. A análise das diretrizes para a educação nacional permite dizer que $o$ direito à diferença é reconhecido como forma de equiparar oportunidades, mas a estrutura e o modus operandi desse trabalho ainda são frágeis e insuficientes para atender às diferentes necessidades de apoio desses alunos.

Palavras-chave: deficiência; educação inclusiva; perspectiva multidimensional; necessidades de apoio; políticas públicas. 


\begin{abstract}
The article discusses the concepts of disability expressed in reference documents of today and their implications for the school, based on a reflection on the process of affirming the rights of persons with disabilities. The multidimensional perspective focuses on relations between the individual aspects and the environment where the disabled person is inserted, resulting in an approach that emphasizes functional difficulties, so as to encourage the provision of services and support to meet the specific demands of each person. This perspective has implications in the design and implementation of public policies in several areas, and also in education. Analysis of guidelines for national education law allow us to say that the right to be different is recognized as a way to equalize opportunities, but the structure and way of working to meet the support needs of different students is still fragile and insufficient.

Keywords: disability; inclusive education; multidimensional perspective; support needs; public policies.
\end{abstract}

Na perspectiva da educação inclusiva, a função da escola é compreendida também como um espaço de aprendizagem de valores essenciais à vida com dignidade, tais como a solidariedade, a compreensão mútua, o diálogo e o senso de justiça, entre outros aspectos do convívio social. Por isso, a prática da educação inclusiva diz respeito a uma decisão ética sobre a formação que queremos oferecer às novas gerações, tendo como horizonte a sociedade que queremos construir, nosso sonho de futuro.

Sentir-se parte da vida em sociedade, em todas as suas dimensões, é o que todo ser humano deseja. Segundo a Organização Mundial da Saúde (OMS), a qualidade de vida está relacionada à "percepção do indivíduo de sua posição na vida no contexto da cultura e sistema de valores nos quais ele vive e em relação aos seus objetivos, expectativas, padrões e preocupações" (The WHOQOL Group, 1995 apud FLECK, 2000, p. 34). Mas, no caso das pessoas com deficiência, considerando que, ao longo da história da humanidade estiveram quase sempre privadas de ocupar determinadas posições de destaque na sociedade, de ter expectativas em relação ao futuro, como qualquer outra pessoa, porque foram excluídas e segregadas, esta percepção pode se tornar bastante alterada. Muitas dessas pessoas ainda desconhecem seus direitos, outras não sabem como exigi-los e há 
também aquelas que assimilaram uma concepção assistencialista e entendem o direito como favor ou privilégio.

Nas escolas, é muito comum que os pais aceitem passivamente algumas exigências para que seus filhos com deficiência frequentem a sala de aula comum, sem exigirem recursos e serviços de apoio para garantir a igualdade de oportunidade no processo de aprendizagem. Isso ocorre porque é bastante frequente que pais e profissionais de educação ignorem a legislação que protege os direitos dos alunos com deficiência, até porque, historicamente, esses direitos nem sempre foram reconhecidos.

O século XXI trouxe grandes avanços na afirmação dos direitos das pessoas com deficiência e cada vez ganha mais relevância a compreensão de que a deficiência é multidimensional, sendo necessário levar em consideração tanto os aspectos biológicos, quanto os sociais e psicológicos de cada indivíduo para oferecer melhores condições de vida para todos. Vivemos, portanto, um momento de mudança nas concepções sobre deficiência e nos modos de se relacionar, conviver e garantir condições de acesso e participação a essas pessoas.

No Brasil, as ações afirmativas, em geral, garantem o acesso às pessoas com deficiência na escola regular, no mercado de trabalho e nos espaços sociais. Mas as necessidades de apoio para garantir acesso com qualidade e participação real são desafios que precisam ser contemplados na elaboração e implementação de novas políticas públicas. Por isso, assume importância a discussão sobre os conceitos atuais de deficiência e seus impactos na construção de uma consciência coletiva sobre a necessidade de se enfrentar este problema.

Ao longo da história da humanidade, a deficiência foi compreendida de muitas formas diferentes, em função de uma multiplicidade de fatores, que abarcam desde o conhecimento científico disponível e socializado até os valores intrínsecos ao modo como cada sociedade se organiza e funciona. Durante séculos, as pessoas com deficiência foram compreendidas como anormais, e não havia distinção clara entre as limitações de ordem sensorial, física ou intelectual, pois todos eram categorizados como desvios da normalidade (JANUZZI, 1992; BUENO, 1993). Consequentemente, a identidade dessas pessoas era construída prioritariamente pelo viés da deficiência, o que significava ocupar o lugar social de anormal, retardado, aleijado, deficiente, entre outros adjetivos comumente atribuídos a elas. Esta noção de 
anormalidade sugere impossibilidade ou incapacidade para o convívio social, a aprendizagem escolar e a vida profissional, como se a deficiência fosse um atributo unicamente pessoal.

Remonta ao século XX o reconhecimento da especificidade da deficiência mental, hoje também denominada deficiência intelectual, embora a compreensão desses conceitos ainda seja bastante diversa. Segundo Jannuzzi (1992), foi em 1939, no Congresso de Genebra, que a expressão deficiente mental surgiu como tentativa de padronizar a forma de se referir às pessoas nessa condição e substituir o termo anormal, que até então era usualmente utilizado para se referir a todas as pessoas com algum tipo de deficiência, como já mencionado. Em relação à expressão deficiência intelectual, de acordo com Sassaki (2005), seu uso foi legitimado em 2004 pela Declaração de Montreal sobre a Deficiência Intelectual. Esta mudança de nomenclatura foi proposta com a intenção de distinguir o funcionamento da mente, como um todo, do funcionamento do intelecto, pois é frequente a indistinção entre doença mental e deficiência mental.

Foi também a partir século XX, com os avanços da neurociência, que ficou patente a capacidade e a potencialidade das pessoas com deficiências. Ainda no final do século XIX surgiram os primeiros estudos sobre a capacidade de reorganização do cérebro, mas apenas em 1940 a reorganização neural seria chamada, pela primeira vez, de plasticidade cerebral (ROTTA, 2006). Segundo Oda, Santana e Carvalho (2002, p. 173), o conceito de plasticidade cerebral "se refere à adaptação que experimenta o sistema nervoso diante de mudanças em seu meio externo e interno, além disso, pode refletir a adaptação funcional do cérebro para minimizar os efeitos das lesões estruturais e funcionais". Esses autores, assim como Rotta (2006), Consenza e Guerra (2011), enfatizam que a plasticidade ocorre ao longo da vida, ou seja, que até mesmo o cérebro de adultos tem capacidade de se reorganizar para atender às demandas funcionais. Os mesmos autores também ressaltam que o ambiente tem relação direta com a plasticidade cerebral e, consequentemente, com a aprendizagem, pois as modificações do sistema nervoso central ocorrem a partir dos estímulos e/ou experiências de vida dos indivíduos.

Nesse contexto, atualmente ganha força a concepção social de deficiência, que coloca em foco a relação entre os aspectos individuais e o ambiente no qual a pessoa esteja inserida, considerando o desempenho como resultado de múltiplas variáveis e não apenas a expressão de uma condição pessoal. Esse entendimento da condição de deficiência está expresso em três 
documentos internacionais que foram elaborados no século atual.

0 primeiro deles é a Classificação Internacional de Funcionalidade, Incapacidade e Saúde (CIF), que foi elaborada em 2001 pela Organização Mundial da Saúde e contribuiu sobremaneira para a ressignificação do conceito de deficiência. Segundo Farias e Buchalla (2005, p. 190),

\section{(...) os conceitos apresentados na classificação introduzem um novo paradigma para pensar e trabalhar a deficiência e a incapacidade: elas não são apenas uma consequência das condições de saúde/doença, mas são determinadas também pelo contexto do meio ambiente físico e social, pelas diferentes percepções culturais e atitudes em relação à deficiência, pela disponibilidade de serviços e de legislação.}

De acordo com a CIF, "a funcionalidade de um indivíduo num domínio específico é uma interação ou relação complexa entre a condição de saúde e os fatores contextuais (fatores ambientais e pessoais)" (ORGANIZAÇÃO MUNDIAL DA SAÚDE, 2004, p. 20, tradução nossa). Essa funcionalidade está representada na figura 1 a seguir:

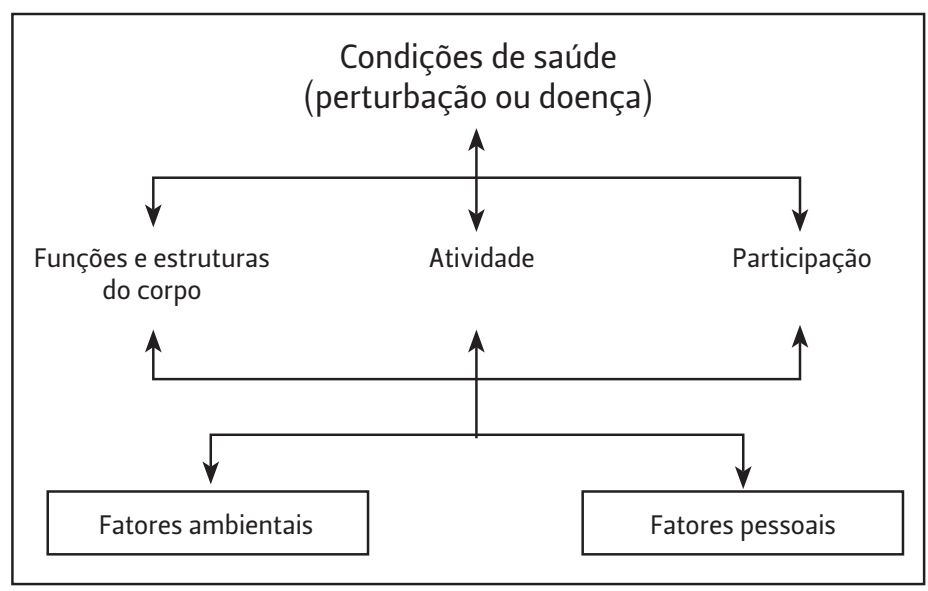

Figura 1 - Interações entre os componentes da CIF Fonte: OMS (2004, p. 20, tradução nossa).

Nessa abordagem multidimensional, o que realmente importa é a descrição da funcionalidade e da incapacidade ${ }^{1}$ decorrentes de várias possibilidades de interação entre as diferentes dimensões. Por exemplo, uma pessoa pode:

- ter deficiência sem limitações de capacidade (ex: uma desfiguração resultante da Doença de Hansen pode não ter efeito sobre a capacidade da pessoa);

- ter problemas de desempenho e limitações de capacidade

1. Na CIF (ORGANIZAÇÃO MUNDIAL DA SAÚDE, 2004), o termo incapacidade "inclui deficiências, limitação da atividade ou restrição na participação" (p. 7) e é caracterizado "como o resultado de uma relação complexa entre a condição de saúde do indivíduo e os fatores pessoais, com os fatores externos que representam as circunstâncias nas quais o indivíduo vive (p. 19, tradução nossa). 
sem deficiências evidentes (ex: redução de desempenho nas atividades diárias associado a várias doenças);

- ter problemas de desempenho sem deficiências ou limitações de capacidade (ex: indivíduo HIV positivo ou exdoente curado de doença mental que enfrenta estigmas ou discriminações nas relações interpessoais ou no trabalho);

- ter limitações de capacidade se não tiver assistência, e nenhum problema de desempenho no ambiente habitual (ex: um indivíduo com limitações de mobilidade pode se beneficiar de ajudas tecnológicas de assistência para se movimentar);

- experimentar um grau de influência em sentido contrário (ex: a inatividade dos membros pode levar à atrofia muscular; a institucionalização pode resultar numa perda da socialização). (ORGANIZAÇÃO MUNDIAL DA SAÚDE, 2004, p. 20, tradução nossa)

O segundo documento de relevância internacional é o texto da Convenção Internacional dos Direitos da Pessoa com Deficiência (CDPD), que é um tratado aprovado pela Assembleia Geral das Nações Unidas (ONU) em 2006 e ratificado por mais de 100 países, incluindo o Brasil, que em 2008 promulgou o Decreto Legislativo $n^{0} 186 / 08$, aprovando a Convenção e seu protocolo facultativo. Em seu Art. $1^{\circ}$, a deficiência é definida como:

\footnotetext{
Pessoas com deficiência são aquelas que têm impedimentos de longo prazo de natureza física, mental, intelectual ou sensorial, os quais, em interação com diversas barreiras, podem obstruir sua participação plena e efetiva na sociedade em igualdades de condições com as demais pessoas. (BRASIL, 2008).
}

Também na CDPD (2006) a deficiência não é compreendida como um atributo da pessoa, e segundo Lopes (2007, p. 62):

\begin{abstract}
A principal contribuição desse tratado internacional é a positivação da mudança do paradigma da visão da deficiência no mundo, que passa do modelo médico e assistencialista, no qual a deficiência é tratada como um problema de saúde, para o modelo social de direitos humanos, no qual a deficiência é resultado da equação de interação da limitação funcional com o meio. Essa transformação deve mudar o planejamento das políticas públicas do mundo inteiro.
\end{abstract}

0 mais recente documento de expressão internacional é o Relatório Mundial sobre a Deficiência (World Report on Disability), 
publicado pela OMS em 2011, o qual identifica a deficiência como "complexa, dinâmica, multidimensional e questionada" e reconhece que "o ambiente de uma pessoa tem um enorme impacto sobre a experiência e a extensão da deficiência" (ORGANIZAÇÃO MUNDIAL DA SAÚDE, 2011, p. 4). 0 mesmo relatório afirma que, "embora sem a intenção de discriminar, indiretamente o sistema exclui as pessoas com deficiência ao não levar em conta suas necessidades" (p. 6).

Essa compreensão da deficiência na perspectiva multidimensional tem implicações diretas para a elaboração e implementação de políticas públicas, pois até então a deficiência era entendida como uma condição de saúde imutável, que não tinha relação direta com os aspectos ambientais. Portanto, a condição da pessoa com deficiência não era vista como uma construção social. Já na abordagem proposta pela OMS, a sociedade e o Estado são responsabilizados pela provisão de serviços e recursos de apoio que permitam maior atividade e participação social.

Segundo Secchi (2010), as políticas públicas buscam soluções para problemas considerados relevantes para a coletividade em relação a situações consideradas inadequadas, uma vez que tais situações não atendem ao que é desejado e idealizado como possível. Este autor chama a atenção para o fato de que

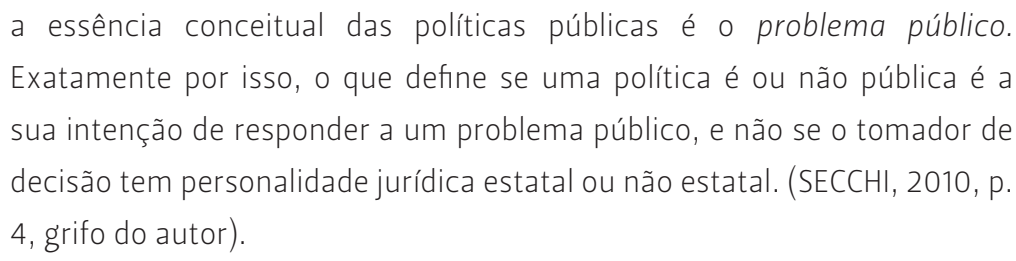

De acordo com essa abordagem, alguns aspectos da vida de pessoas com deficiência já são reconhecidos como problemas pela coletividade e, por isso, há uma série de decisões e ações, nos âmbitos legislativo e executivo, que buscam oferecer melhores condições de vida a elas. Um exemplo é a Lei ${ }^{\circ}$ 8.213/91, também conhecida como Lei de Cotas, que instituiu a obrigatoriedade das empresas reservarem uma porcentagem de postos de trabalho às pessoas com deficiência (BRASIL, 1991). Esta lei é uma ação afirmativa em prol do direito ao trabalho e significa o reconhecimento de que a maioria das pessoas com deficiência pode ser produtiva. Assim como essa lei, na legislação brasileira há outros dispositivos normativos que garantem às pessoas com deficiência o direito de frequentar a classe comum da escola regular, ter atendimento educacional especializado complementar 
ou suplementar, isenção tarifária em transportes públicos, entre outros.

No entanto, as discussões mais recentes sobre deficiência apontam como principal problema a ausência e/ou insuficiência de serviços de assistência e apoios específicos (suportes) para atender às necessidades das pessoas com deficiência, incapacidade ou mobilidade reduzida de modo a garantir funcionalidade, visando autonomia, independência e qualidade de vida.

O Relatório Mundial sobre a Deficiência (OMS, 2011) aponta que "são escassas as evidências sobre a demanda e oferta de serviços de suporte e assistência, mesmo em países desenvolvidos" (ORGANIZAÇÃO MUNDIAL DA SAÚDE, 2011, p. 145), mas enfatiza:

\section{[...] muitas pessoas com deficiência precisam de assistência e suporte para alcançar uma boa qualidade de vida e serem capazes de participar da vida econômica e social em igualdade de condições com as demais pessoas. [...] A maior parte da assistência e do suporte vem de membros da família ou de redes sociais. 0 fornecimento de serviços formais por parte do Estado é, de forma geral, insuficiente, as organizações sem fins lucrativos têm abrangência limitada, e organizações privadas raramente oferecem um suporte economicamente viável às necessidades das pessoas com deficiência. (p. 143).}

No Brasil, o atendimento às necessidades das pessoas com deficiência ainda é um desafio. 0 acesso a serviços específicos de apoio, por parte das pessoas com deficiências, para garantir a igualdade de oportunidades nas diferentes dimensões na vida social, como educação, saúde, trabalho, lazer, esporte, cultura, turismo, transporte ou assistência social, ainda não é compreendido como um problema público, ou seja, como um problema reconhecido pela coletividade como relevante.

Portanto, na atualidade, mais importante do que uma "abordagem para a deficiência", é utilizarmos uma "abordagem para as dificuldades funcionais" (ORGANIZAÇÃO MUNDIAL DA SAÚDE, 2011, p. 47), pois a construção desse entendimento favorecerá a implementação de ações no sentido de ofertar serviços específicos para atender às demandas de apoio de pessoas com deficiência, incapacidade ou mobilidade reduzida. Nesse contexto, cabe refletir sobre como as escolas estão atendendo às necessidades de apoio dos alunos com deficiência e oferecendo recursos, metodologias, estratégias e serviços que ampliem as habilidades funcionais dos mesmos, criando condições mais favoráveis ao seu aprendizado e desenvolvimento. 
Uma concepção de educação que contempla as diferenças concebe o ensino como resposta às necessidades e estilos de aprendizagem de todos os alunos, valorizando a interação entre eles, a participação ativa, a autonomia e a colaboração nas situações de ensino. É, nesse sentido, uma abordagem que busca equilíbrio entre as necessidades de igualar e diferenciar para garantir o direito a uma educação de qualidade para todo e qualquer aluno.

Muitas vezes é necessário garantir condições iguais a todos os alunos, tais como condições de acesso aos espaços da escola, de participação em eventos e saídas pedagógicas etc. Em outras situações, o mais importante é diferenciar para garantir que todos os alunos possam desfrutar de situações desafiadoras, que resultem em participação e aprendizagem. Para isso, é necessário identificar qual é a barreira que está dificultando o aprendizado, ou a participação, e propor recursos, estratégias ou serviços que potencializem a capacidade de aprender em sala de aula. Trata-se de oferecer apoios, que muitas vezes são soluções simples, como por exemplo:

- para facilitar a leitura de um texto, alunos com baixa visão podem utilizar uma lupa, e alunos com deficiência física podem dispor de um cavalete para fixar as folhas verticalmente;

- e para a apoiar a produção textual de alunos com deficiência intelectual é possível construir um roteiro com a sequência de ideias a ser escrita.

Enfim, são muitas as formas de diferenciar para garantir o direito à educação a todos os alunos, e não há um manual que possa ser oferecido aos professores e às escolas que dê conta da complexidade e da diversidade do ser humano, e, portanto, do grupo de alunos e de cada um em particular.

De acordo com o Decreto $n^{0} 7.611 / 2011$ (BRASIL, 2011), que estabelece diretrizes para a educação especial no país, um dos objetivos dessa modalidade escolar é a "adoção de medidas de apoio individualizadas e efetivas, em ambientes que maximizem o desenvolvimento acadêmico e social, de acordo com a meta de inclusão plena" (Art. $1^{\circ}$, Inciso VI). Portanto, as diretrizes oficiais para a educação orientam os sistemas de ensino a oferecer apoio aos alunos com deficiência, pois reconhecem o direito à diferença como direito à equiparação de oportunidades para alcançar boas condições de desenvolvimento para todos os alunos. 
O Atendimento Educacional Especializado (AEE) previsto nos documentos oficiais do Ministério da Educação é uma forma de atender às diferentes necessidades de apoio dos alunos que são público-alvo da Educação Especial'2. Segundo a Resolução CNE/CEB $n^{\circ}$ 4/2009:

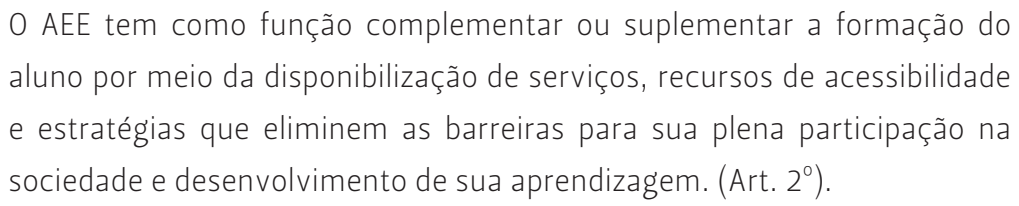

No entanto, para que tais diretrizes se efetivem nas escolas, é preciso pensar a atuação da educação especial em diferentes abordagens - em salas multifuncionais, no contraturno, na sala de aula comum em parceria com o professor da classe, em centros especializados e, enfim, em formatos variados -, de modo que se possa atender à diversidade de demandas desses alunos.

Numa perspectiva multidimensional, também é indispensável uma avaliação criteriosa das necessidades de apoio dos alunos, o que permite planificar os serviços, as estratégias e os suportes necessários para cada criança ou jovem usufruir das situações de ensino da melhor forma possível. Mas esta avaliação vai muito além do diagnóstico clínico, pois requer uma avaliação descritiva das dificuldades funcionais que devem ser foco da equipe que trabalha com cada um dos alunos.

Um aspecto que merece destaque é que em nenhum dos documentos normativos da educação especial há menção à exigência de avaliações diagnósticas clínicas para que alunos com deficiência recebam os apoios necessários. Ao contrário, em 2006, o documento intitulado Saberes e práticas da inclusão: avaliação para identificação das necessidades educacionais especiais apresenta críticas a uma prática instituída em muitas escolas brasileiras, na qual o laudo (diagnóstico clínico) é uma exigência para a inserção em serviços especializados de educação:

\footnotetext{
Os próprios integrantes de equipes de diagnóstico, geralmente composta por psicólogos, pedagogos e fonoaudiólogos, declaram-se insatisfeitos com suas práticas, pois reconhecem que são pautadas numa visão estática do quadro em que se encontra o aluno avaliado. Sabem, ainda, que os laudos funcionam como "etiquetas" que rotulam, discriminam e segregam. E, o que é mais sério, pouco têm servido para orientar o trabalho pedagógico a ser desenvolvido pelos professores [...] (BRASIL, 2006, p. 86)
}

2. Alunos com deficiência, transtorno global do desenvolvimento e altas habilidades/superdotação. 
A perspectiva multidimensional requer uma equipe multidisciplinar que trabalhe de forma complementar, pois as necessidades de apoio podem se relacionar a diferentes áreas: pedagogia, psicologia, fonoaudiologia, terapia ocupacional, assistência social, entre outras. Se trabalharem de forma isolada, esses profissionais terão dificuldade para conseguir resultados efetivos. Também as áreas da medicina - neurologia, genética, psiquiatria, entre outras - podem ser contributivas para se estabelecer um plano de apoio adequado às necessidades dos alunos. Essa complementaridade é enriquecedora tanto do ponto de vista da avaliação das necessidades de apoio, como do planejamento do trabalho a ser feito com cada aluno, e também na implementação dos apoios e serviços especializados.

No Brasil, o trabalho pedagógico, de modo geral, não acontece em parceria com profissionais de diferentes áreas, o que se constitui em um desafio a ser superado. Embora 0 direito à diferença seja reconhecido como forma de equiparar oportunidades, a estrutura e a forma de trabalho ainda são frágeis e insuficientes para atender às diferentes necessidades de apoio dos alunos.

Vivemos hoje um momento em que é preciso reinventar, inovar e ousar para construir um modelo de educação e de sociedade que resguarde o que é individual e singular, e proteja o que é comum e compartilhado, possibilitando formas de convivência enriquecedoras e pacíficas.

\section{REFERÊNCIAS}

BRASIL. Lei n 8.213, de 24 de julho de 1991. Dispõe sobre os Planos de Benefícios da Previdência Social e dá outras providências. Diário Oficial da União, Brasília, 25 jul 1991. Disponível em: <http://www.planalto.gov.br/ccivil_03/leis/ L8213cons.htm>. Acesso em: 27 mar. 2012.

Saberes e práticas da inclusão: avaliação para identificação das necessidades educacionais especiais. 2. ed. Brasília: MEC/SEESP, 2006. (Série: Saberes e práticas da inclusão) 
Disponível em: <http://www. planetaeducacao.com.br/portal/ documentos_apoio/convencao-sobre-os-direitos-das-pessoascom-deficiencia-comentada.pdf>. Acesso em: 10 abr. 2013.

Resolução CEB/CNE n 4, de 2 de outubro de 2009. Institui Diretrizes Operacionais para o Atendimento Educacional Especializado na Educação Básica, modalidade Educação Especial. Diário Oficial da União, Brasília, 2009. Disponível em: <http://portal.mec.gov.br/dmdocuments/ rceb004_09.pdf>. Acesso em: 25 jan. 2010.

Decreto 7.611/2011, de 17 de novembro de 2011. Dispõe sobre a educação especial, o atendimento educacional especializado e dá outras providências. Diário Oficial da União, Brasília, 2011. Disponível em: <http://www.planalto.gov.br/ ccivil_03/_Ato2011-2014/2011/Decreto/D7611.htm>. Acesso em: $21 \mathrm{fev} .2013$.

BUENO, José Geraldo Silveira. Educação especial brasileira: integração/segregação do aluno diferente. São Paulo: EDUC, 1993.

CONSENZA, Ramon M.; GUERRA, Leonor B. Neurociência e educação: como o cérebro aprende. Porto Alegre: Artmed, 2011.

FARIAS, Norma; BUCHALLA, Cassia Maria. A classificação internacional de funcionalidade, incapacidade e saúde da organização mundial da saúde: conceitos, usos e perspectivas. Revista Brasileira de Epidemiologia, São Paulo, v. 8, n. 2, p. 187-193, jun. 2005. Disponível em: <http://www. scielo.br/pdf/rbepid/v8n2/11.pdf>. Acesso em: 10 abr. 2013.

FLECK, Marcelo Pio de Almeida. 0 instrumento de avaliação de qualidade de vida da Organização Mundial da Saúde (WHOQOL-100): características e perspectivas. Ciência \& Saúde Coletiva, Rio de Janeiro, v. 5, n. 1, p. 33-38, 2000. Disponível em: <http://www.scielo.br/pdf/csc/v5n1/7077.pdf > Acesso em: 3 out. 2012.

JANNUZZI, Gilberta. A luta pela educação do deficiente mental no Brasil. Campinas: Autores Associados, 1992.

LOPES, Laís V. C. de Figueiredo. Convenção sobre os direitos da pessoa com deficiência da ONU. In: GUGEL, Maria Aparecida; COSTA FILHO, Waldir Macieira da; RIBEIRO, Lauro Luiz Gomes. Deficiência no Brasil: uma abordagem integral dos direitos das 
pessoas com deficiência. Florianópolis: Obra Jurídica, 2007. p. 4165.

ODA, Juliano Yasuo; SANTANA, Débora de Mello Gonçales;

CARVALHO, Jaqueline de. Plasticidade e regeneração funcional do sistema nervoso: contribuição ao estudo de revisão. Arquivos de Ciências da Saúde da UNIPAR, v. 6, n. 2, p. 171-176, 2002. Disponível em: <http://revistas.unipar.br/saude/article/ view/1175/1037>. Acesso em: 10 abr. 2013.

ORGANIZAÇÃO MUNDIAL DA SAÚDE. Classificação Internacional de Funcionalidade, Incapacidade e Saúde. Lisboa: Direcção-Geral da Saúde, 2004. Disponível em: <http://www.inr.pt/uploads/docs/ cif/CIF_port_\%202004.pdf>. Acesso em: 25 jan. 2010.

Relatório Mundial sobre a Deficiência. Tradução de Lexicus Serviços Linguísticos. São Paulo: SEDPcD, 2011.

ROTTA, Newra Tellecchea. Plasticidade cerebral e aprendizagem. In: ROTTA, Newra Tellecchea; OHLWEILER, Lygia; RIESGO, Rudimar dos Santos (Orgs.). Transtornos da aprendizagem: abordagem neurobiológica e multidisciplinar. Porto Alegre: Artmed, 2006. p. 453-472.

SASSAKI, Romeu. Deficiência mental ou intelectual? Doença ou transtorno mental? Atualizações semânticas na inclusão de pessoas. Revista Nacional de Reabilitação, São Paulo, ano IX, n. 43, p. 9-10, mar./abr. 2005. Disponível em: <http://www.escoladegente.org.br/ noticiaDestaque.php?id=452>. Acesso em: 26 mar. 2012.

SECCHI, Leonardo. Políticas públicas: conceitos, esquemas de análise, casos práticos. São Paulo: Cengage Learning, 2010.

Recebido em: 22/02/2013

Revisado em: 03/03/2013

Aprovado em: 05/03/2013 